\title{
Factores clínicos y demográficos predictores de éxito del misoprostol en el tratamiento médico del aborto espontáneo
}

\author{
Marta Camacho C., PhD, Josefa Quesada H., PhD, María José Palomo V., PhD, \\ Eva María Sánchez S., Luis Miguel Cóndor M., PhD.
}

Facultad de Medicina de Málaga, Hospital Universitario Virgen de la Victoria, Málaga, España.

\section{RESUMEN}

Objetivos: Determinar posibles predictores de éxito del misoprostol en el tratamiento del aborto espontáneo del primer trimestre. Método: Estudio observacional descriptivo y prospectivo, realizado entre febrero de 2009 y febrero de 2010. Inclusión consecutiva de 248 mujeres con diagnostico ecográfico de aborto espontáneo del primer trimestre con tratamiento médico o quirúrgico, siendo las pacientes las que eligieron la opción terapéutica de acuerdo a los criterios de inclusión para el manejo con misoprostol. En el grupo tratamiento médico se aplicó $800 \mathrm{mcg}$ de misoprostol vaginal/24horas/2 dosis, considerándose como criterio de éxito un endometrio homogéneo con grosor $\leq 15 \mathrm{~mm}$ en la ecografía realizada al $8^{\circ}$ día del tratamiento. Resultados: Influyen en la tasa de éxito del misoprostol la edad de las pacientes (mejor resultado cuanto más joven, $p=0,025$ ), número de embarazos (responden mejor las primigestas, $p=0,024$ ), existencia o no de abortos $(p=0,05)$ o legrados previos $(p=0,028)$ (la tasa de éxito del misoprostol es mayor en las mujeres que no tienen ningún aborto o legrado previo), y tipo de sangrado vaginal que aparece como efecto secundario del misoprostol (mejorando el pronóstico cuando dicho sangrado es igual o mayor que menstruación, $\mathrm{p}=0,041)$. Conclusiones: Hubo predictores de éxito del misoprostol que pueden orientar el manejo, sabiendo que hubo mejor resultado en pacientes jóvenes, primigestas, sin abortos ni legrados previos y con un sangrado vaginal igual o mayor que menstruación.

\section{PALABRAS CLAVE: Misoprostol, aborto espontáneo, legrado, éxito de tratamiento}

\section{SUMMARY}

Objectives: To determine possible predictors of success of misoprostol in the treatment of first trimester spontaneous abortion. Methods: Descriptive observational study and prospectively from February 2009 to February 2010. It were included 248 women which were diagnosed by ultrasound of spontaneous abortion in the first trimester and received medical or surgical treatment, depending on the patient's own choice, provided that the established clinical conditions were present. The protocol applied in the medical treatment group was $800 \mathrm{mcg}$ of vaginal misoprostol/24h/2 dose. It was considered as criteria of success, the presence of a homogeneous endometrium with a thickness $\leq 15 \mathrm{~mm}$ in the ultrasound examination performed on the 8 th day of treatment. Results: The following variables influence the success rate of misoprostol: patient age (the younger the better outcome, $p=0.025$ ), number of pregnancies (primiparous respond better, $p=$ $0.024)$, presence or absence of abortions $(p=0.05)$ or previous curettage $(p=0.028)$ (the success rate of misoprostol is higher in women who have no previous abortion or curettage), and type of vaginal bleeding 
that occurs as a side effect of misoprostol (improving prognosis when bleeding is equal to or greater than the rule, $p=0.041$ ). Conclusions: We found predictors of success of misoprostol, which can guide the management knowing that better results can get obtained in younger patients, primigravida, no previous abortions or curettage and with a vaginal bleeding equal to or greater than the rule.

\section{KEY WORDS: Misoprostol, spontaneous abortion, curettage, success of treatment}

\section{INTRODUCCIÓN}

El misoprostol tiene un papel potencial en el tratamiento del aborto espontáneo, ya que hasta la introducción del mismo con tal fin, los clínicos y pacientes sólo podían elegir entre cirugía (legrado) o no hacer nada (conducta expectante).

El tratamiento médico está ganando lentamente importancia constituyendo una alternativa terapéutica altamente aceptable, como un método para la evacuación uterina en las pérdidas reproductivas precoces, fácil de usar, asequible y de bajo costo, que podría revolucionar el tratamiento para esta condición (1).

En los estudios publicados, la tasa de éxito del misoprostol varía del 13 al $100 \%$, con una media de 92\% aproximadamente (2). Este amplio rango de eficacia puede deberse a diferencias en el diseño del estudio, las dosis y vía empleadas, criterio de éxito establecido, tiempo de espera considerado para evaluar el resultado, etc.

El objetivo de esta investigación es comparar la utilidad de diferentes parámetros (demográficos, clínicos y analíticos) para predecir el resultado del manejo médico del aborto espontáneo, y ser capaces de este modo, de seleccionar las pacientes que responden mejor al misoprostol.

\section{PACIENTES Y MÉTODO}

Estudio observacional descriptivo, con carácter prospectivo, aprobado por el Comité de Ética de la Institución. Fueron incluidas todas la pacientes $(n=248)$ diagnosticadas ecográficamente de aborto espontáneo del primer trimestre (del subtipo de aborto diferido, incompleto, gestación anembrionada ó aborto en curso) en el Servicio de Urgencias de Obstetricia y Ginecología del Hospital Universitario Virgen de la Victoria de Málaga, desde febrero de 2009 a febrero de 2010 y que recibieron tratamiento (médico o quirúrgico), comprobando que se ajustaban, en el caso del tratamiento médico, a los siguientes criterios de inclusión y exclusión $(3,4)$ : Criterios de inclusión: Aborto diferido con una longitud cráneo-caudal de hasta $40 \mathrm{~mm}$ (que correspon- de a 10 semanas de gestación), gestación anembrionada hasta un tamaño del saco gestacional de $45 \mathrm{~mm}$, aborto incompleto hasta un tamaño uterino de 12 semanas y un grosor endometrial $>15 \mathrm{~mm}$ y aborto en curso, aplicando las mismas condiciones. Criterios de exclusión: Hemoglobina $<9 \mathrm{~g} / \mathrm{dl}$, trombopenia $<100.000 / \mathrm{mm} 3$, paciente hemodinámicamente inestable, alergia a misoprostol $u$ otra prostaglandina, fiebre, sospecha de endometritis, aborto provocado, aborto en curso o incompleto con sangrado muy abundante, sospecha de gestación molar, fracaso del tratamiento médico con misoprostol y causas personales.

Tras establecer el diagnóstico de aborto espontáneo ecográficamente, se valoró en cada paciente los criterios para el tratamiento con misoprostol anteriormente expuestos y sólo si los cumplía, se le ofertaba las dos posibilidades de tratamiento (médico ó quirúrgico), siendo la propia paciente la que elegía la opción terapéutica. Si no los cumplía, el tratamiento establecido fue el legrado. Para uno u otro tratamiento se procedió a la obtención del consentimiento informado correspondiente.

A las pacientes que optaron por tratamiento con misoprostol, se les aplicó un protocolo sencillo, que requiere sólo dos consultas externas. En la primera consulta se comprueba el diagnóstico ecográfico de aborto espontáneo (descartando una gestación ectópica ó un embarazo molar) y que éste cumple los criterios de inclusión para el tratamiento médico ya reseñados. La segunda consulta, se programa ocho días después de la administración del misoprostol, para confirmar el éxito o no del mismo. Se definió como criterio de éxito del tratamiento médi$\mathrm{co}$, un endometrio homogéneo $\leq 15 \mathrm{~mm}$ a la ultrasonografía (5).

Respecto al tratamiento quirúrgico, se consideró que fue eficaz si la paciente no precisó relegrado durante el mes que siguió al procedimiento (confirmado con control telefónico al mes).

La pauta de administración del misoprostol aplicada en este estudio fue: $800 \mathrm{mcg}$ (4 comprimidos de $200 \mathrm{mcg}$ ) vía vaginal el primer día. Al día siguiente se repite misma dosis a la misma hora (1). La paciente es la que se autoadministra la medicación en 
casa. Se le recomienda humedecer previamente los comprimidos para facilitar la posología, y permanecer en reposo 1-2 horas.

En el análisis estadístico inferencial bivariante, para determinar posibles variables que pudieran estar relacionadas con la tasa de éxito del misoprostol, se asumió un intervalo de confianza del $95 \%$. Se consideró como diferencia significativa valores de $p \leq 0,05$.

Dicho análisis se realizó utilizando el test de la t-student cuando se comparan dos variables de grupos independientes que siguen una distribución normal, ó el test no paramétrico de Mann-Whitney en caso de no cumplirse la normalidad. Para comparar más de dos grupos de una variable cuantitativa se utilizó el Análisis de la Varianza (ANOVA), cuando la variable siguió una distribución normal; en el caso de no cumplirse la normalidad y/o homocedasticidad, se utilizó el test no paramétrico Kruskall-Wallis. Para la relación entre dos variables cualitativas, se utilizó las tablas de contingencia para su descripción y el test Chi-cuadrado como test de independencia/homogeneidad.

\section{RESULTADOS}

En cuanto a las características de la población estudiada, encontramos que ésta fue homogénea en los dos grupos de tratamiento (Tabla I). Esta homogeneidad resultante de forma espontánea, pese a no haber aleatorizada la muestra, nos permitió continuar con el estudio por no haber sesgo.

La tasa de éxito (consecución de aborto completo) obtenida fue del $84,4 \%$ para el misoprostol y el $99,9 \%$ para el legrado.

No hubo diferencias estadísticamente significativas en la tasa de éxito del misoprostol en función del nivel de estudios, grupo sanguíneo-Rh, paridad, número de cesáreas, edad gestacional, volumen de sangrado vaginal objetivado en primera visita y diagnóstico ecográfico establecido en primera visita (Tabla II).

Hubo una relación con significación estadística entre la eficacia del tratamiento médico y las siguientes variables (Tabla III):

Edad de las pacientes: son más jóvenes las del grupo de éxito del misoprostol (en el grupo de éxito la media de edad son 33 años, en el grupo del fracaso 36 años, $p=0,025$ )

Número de embarazos: hubo una tendencia estadísticamente significativa al éxito en las primigestas $y$ al fracaso en las restantes $(p=0,024)$.

Abortos previos: en las pacientes que no tienen aborto anterior, es más frecuente que se dé una buena respuesta al misoprostol $(\mathrm{p}=0,05)$.

Legrados previos: el éxito del tratamiento médico es más frecuente en las pacientes que no tienen legrados previos $(p=0,028)$.

Tabla I

CARACTERÍSTICAS DEMOGRÁFICAS Y CLÍNICAS DE LA POBLACIÓN DE ESTUDIO

\begin{tabular}{llcc}
\hline Variables & & Misoprostol & Legrado \\
\hline Edad & & $33,31(18-47)$ & $32,90(18-45)$ \\
& Universitarios & $48,2 \%$ & $47,1 \%$ \\
Nivel de estudios & Básicos & $41,10 \%$ & $42,6 \%$ \\
& No estudios & $10,7 \%$ & $10,3 \%$ \\
Edad gestacional (semanas) & & $69,53(11-111)$ & $71,12(12-116)$ \\
$N^{\circ}$ embarazos & & $2,13(1-6)$ & $2,04(1-11)$ \\
$N^{\circ}$ partos & & $0,66(0-3)$ & $0,53(0-6)$ \\
$N^{\circ}$ abortos & & $0,43(0-4)$ & $0,40(0-6)$ \\
$N^{\circ}$ legrados & & $0,30(0-3)$ & $0,37(0-6)$ \\
$N^{\circ}$ cesáreas & & $0,70(0-1)$ & $0,11(0-2)$ \\
& $>$ regla & $5,40 \%$ & $10,30 \%$ \\
Volumen sangrado vaginal $1^{\text {a }}$ & = regla & $12,50 \%$ & $11,80 \%$ \\
visita & < regla & $49,10 \%$ & $31,60 \%$ \\
& No sangrado & $33,00 \%$ & $46,30 \%$ \\
Diagnóstico ecográfico & Aborto diferido & $49,10 \%$ & $53,70 \%$ \\
$1^{a}$ visita & Aborto incompleto & $25,00 \%$ & $25,70 \%$ \\
& Gestación anembrionada & $25,90 \%$ & $20,60 \%$ \\
\hline
\end{tabular}


Tabla II

VARIABLES NO RELACIONADAS DE FORMA ESTADÍSTICAMENTE SIGNIFICATIVA CON LA TASA DE ÉXITO DEL MISOPROSTOL EN EL TRATAMIENTO DEL ABORTO ESPONTÁNEO DEL PRIMER TRIMESTRE

\begin{tabular}{|c|c|c|c|c|c|}
\hline Variables & & $\mathrm{n}$ & $\begin{array}{l}\text { Éxito } \\
\text { médico }\end{array}$ & $\begin{array}{l}\text { Fracaso } \\
\text { médico }\end{array}$ & Valor $p$ \\
\hline \multirow[t]{3}{*}{ Nivel de estudios } & Universitarios & 53 & $46(50 \%)$ & $7(41,2 \%)$ & \multirow{3}{*}{$p=0,504$} \\
\hline & Básicos & 45 & $38(41,3 \%)$ & $7(41,2 \%)$ & \\
\hline & No estudios & 11 & $8(8,7 \%)$ & $3(17,6 \%)$ & \\
\hline Edad gestacional & Media (semanas) & 109 & 9,48 & 9,93 & $p=0,874$ \\
\hline \multirow[t]{2}{*}{ Partos previos } & No & 60 & $53(57,6 \%)$ & $7(41,2 \%)$ & \multirow[t]{2}{*}{$p=0,211$} \\
\hline & Sí & 49 & $39(42,4 \%)$ & $10(58,8 \%)$ & \\
\hline Cesáreas previas & $\begin{array}{l}\text { No } \\
\text { Sí }\end{array}$ & $\begin{array}{c}101 \\
8\end{array}$ & $\begin{array}{c}85(92,4 \%) \\
7(7,6 \%)\end{array}$ & $\begin{array}{c}16(94,1 \%) \\
1(5,9 \%)\end{array}$ & $p=0,802$ \\
\hline \multirow{4}{*}{ Volumen sangrado $1^{\mathrm{a}}$ visita } & Mayor que regla & 6 & $6(6,5 \%)$ & $0(0,0 \%)$ & \multirow{4}{*}{$p=0,131$} \\
\hline & Igual que regla & 13 & $13(14,1 \%)$ & $0(0,0 \%)$ & \\
\hline & Menor que regla & 53 & $41(44,6 \%)$ & $12(70,6 \%)$ & \\
\hline & No sangrado & 37 & $32(34,8 \%)$ & $5(29,4 \%)$ & \\
\hline \multirow{3}{*}{$\begin{array}{l}\text { Diagnóstico ecográfico } 1^{a} \\
\text { visita }\end{array}$} & Aborto diferido & 53 & $44(47,8 \%)$ & $9(52,9 \%)$ & \multirow{3}{*}{$p=0,760$} \\
\hline & Aborto incompleto & 27 & $24(26,1 \%)$ & $3(17,6 \%)$ & \\
\hline & Gestación anembrionada & 29 & $24(26,1 \%)$ & $5(29,4 \%)$ & \\
\hline
\end{tabular}

Tabla III

VARIABLES RELACIONADAS DE FORMA ESTADÍSTICAMENTE SIGNIFICATIVA CON LA TASA DE ÉXITO DEL MISOPROSTOL EN EL TRATAMIENTO DEL ABORTO ESPONTÁNEO DEL PRIMER TRIMESTRE

\begin{tabular}{|c|c|c|c|c|c|}
\hline Variables & & $\mathrm{n}$ & $\begin{array}{l}\text { Éxito } \\
\text { médico }\end{array}$ & $\begin{array}{l}\text { Fracaso } \\
\text { médico }\end{array}$ & Valor $p$ \\
\hline \multirow[t]{2}{*}{ Edad } & Media (años) & 109 & 33,21 & 36,82 & \multirow[t]{2}{*}{$\mathrm{p}=0,025$} \\
\hline & 1 & 44 & $42(45,7 \%)$ & $2(11,8 \%)$ & \\
\hline $\mathrm{N}^{\circ}$ embarazos & $\begin{array}{l}2 \\
3 \text { ó mas }\end{array}$ & $\begin{array}{l}34 \\
31\end{array}$ & $\begin{array}{l}25(27,2 \%) \\
25(27,2 \%)\end{array}$ & $\begin{array}{l}9(52,9 \%) \\
6(35,3 \%)\end{array}$ & $p=0,024$ \\
\hline Abortos previos & $\begin{array}{l}\text { No } \\
\text { Sí }\end{array}$ & $\begin{array}{l}76 \\
33\end{array}$ & $\begin{array}{l}69(75 \%) \\
23(25 \%)\end{array}$ & $\begin{array}{l}7(41,2 \%) \\
10(58,8 \%)\end{array}$ & $p=0,05$ \\
\hline Legrados previos & $\begin{array}{l}\text { No } \\
\text { Sí }\end{array}$ & $\begin{array}{l}81 \\
28\end{array}$ & $\begin{array}{l}72(78,3 \%) \\
20(21,7 \%)\end{array}$ & $\begin{array}{l}9(52,9 \%) \\
8(47,1 \%)\end{array}$ & $p=0,028$ \\
\hline $\begin{array}{l}\text { Tipo de sangrado } \\
\text { vaginal (efecto } 2^{\circ} \text { ) }\end{array}$ & $\begin{array}{l}\text { Mayor que regla } \\
\text { Igual que regla } \\
\text { Menor que regla }\end{array}$ & $\begin{array}{l}24 \\
45 \\
38\end{array}$ & $\begin{array}{l}21(23,1 \%) \\
42(46,2 \%) \\
28(30,8 \%)\end{array}$ & $\begin{array}{l}3(18,8 \%) \\
3(18,8 \%) \\
10(62,5 \%)\end{array}$ & $p=0,041$ \\
\hline
\end{tabular}


Magnitud del sangrado: el misoprostol es eficaz con mayor frecuencia en las pacientes que refieren un sangrado vaginal igual o mayor que menstruación como efecto secundario del mismo, al contrario cuando el sangrado es menor que menstruación $(p=0,041)$.

\section{DISCUSIÓN}

Con respecto a las variables que según nuestro estudio no se relacionan de forma estadísticamente significativa con el éxito del misoprostol para el tratamiento del aborto espontáneo, encontramos resultados discordantes en la literatura, ya que hay ensayos con una mayor potencia, que sí detectan una relación que alcance significación estadística.

Así, con respecto al grupo sanguíneo y Rh, Mitchell y cols (3) hallan que el factor Rh negativo se asocia a éxito con más frecuencia, observación que no está previamente registrada en la bibliografía, y sobre la que conjeturan que el ser Rh negativa podría ser una razón de fondo para el fracaso del embarazo temprano y crear una situación en la que el misoprostol sea más eficaz. Sin embargo, debido a la falta de una explicación científica, estos autores recomiendan la espera de más pruebas antes de asumir esta asociación como definitivamente verdadera.

En nuestro análisis no detectamos la existencia de una relación que alcance significación estadística, entre el diagnóstico ecográfico establecido en el momento de inclusión de las pacientes y la eficacia del misoprostol, hay estudios donde sí la hay, como sucede en el ensayo randomizado que llevan a cabo Zhang y cols (6), donde se pone de manifiesto que las pacientes con una gestación anembrionada tienen una tasa de éxito del tratamiento médico menor que los otros subtipos de aborto espontáneo (93\% de éxito en las pacientes con aborto incompleto, $88 \%$ en el caso de aborto diferido y $81 \%$ en aquellas con huevo no embrionado, $p=0,02$ ).

Centrándonos en las asociaciones significativas desde el punto de vista estadístico observadas en nuestros resultados, revisando la bibliografía existente, encontramos estudios que coinciden con parte de nuestros hallazgos. Atendiendo al número de embarazos, de la misma manera que nosotros apreciamos una tendencia al éxito $(p=0,024)$ en las primigestas y al fracaso en las restantes, Odeh y cols (7) detectan que un número mayor de embarazos se asocia negativamente con la eficacia del tratamiento médico en el manejo de las pérdidas reproductivas precoces; una asociación negativa que ya fue previamente constatada por Ashok y cols (8) en su ensayo sobre el aborto inducido usando la combinación mifepristona/misoprostol, o por Creinin y cols (5) los cuales sí se centran en el aborto espontáneo y usan sólo misoprostol como nosotros, y proponen como hipótesis explicativa de este hallazgo la posibilidad de que la receptividad del útero a los efectos de este fármaco cambie con la distensión o crecimiento del miometrio en embarazos anteriores.

Por último, como se pone de manifiesto en un ensayo randomizado multicéntrico (9), que evalúa factores clínicos predictores de éxito del misoprostol, éste es más frecuente en las pacientes que relatan un sangrado igual o mayor que menstruación como efecto secundario del fármaco, hallazgo que está en concordancia con nuestro estudio. En cambio, no sucede así con el dolor en hipogastrio, que también lo considera como dato clínico predictivo de eficacia, sin embargo, esta asociación no se da en nuestra investigación.

\section{CONCLUSIÓN}

La información presentada en este análisis puede orientarnos para seleccionar las pacientes en las que es más probable que el tratamiento médico del aborto espontáneo sea eficaz, siendo el perfil ideal una mujer joven, primigesta, sin abortos ni legrados previos; no obstante, no debemos olvidar que no son indicadores definitivos y que la posibilidad de que la paciente sea la que elije el tratamiento, siempre que las condiciones clínicas lo permitan, mejora los resultados en cuanto a calidad de vida (10).

\section{REFERENCIAS}

1. Blum J, Winikoff B, Gemzell-Danielsson K, Ho PC, Schiavon R, Weeks A. A treatment of incomplete abortion and miscarriage with misoprostol. Int J Gynaecol Obstet 2007; 99(Suppl 2):S186-9.

2. Faundes A, Guilherme J, Conde A, Escobedo J, Rizzi $R$, Tavara L, et al. Uso de misoprostol en Obstetricia y Ginecología. FLASOG (Federación Latino americana de Sociedades de Obstetricia y Ginecología). $2^{\underline{a}}$ edición, Marzo 2007; 77-89.

3. Mitchell D, Creinin MD, Xiangke Huang, Carolyn Westhoff, et al. Factors related to successful misoprostol treatment for early pregnancy failure. Obstet Gynecol 2006;107:901-7.

4. Nielsen S, Hanlin M, Platz-Christensen J. Randomised trial comparing expectant with medical management for first trimester miscarriages. Br J Obstet Gynaecol 1999;106:804-7.

5. Creinin MD, Harwood B, Guido RS, Fox MC, Zahang J. Endometrial thickness after misoprostol use for early pregnancy failure. Int J Gynaecol Obstet 2004;86:22-6.

6. Zhang J, Gilles JM, Barnhart K, Creinin MD, Westhoff C, Frederick MM; National Institute of Child Health Human Development (NICHD) Management of Early 
Pregnancy Failure Trial. A comparison of medical management with misoprostol and surgical management for early pregnancy failure. $\mathrm{N}$ Engl J Med 2005;353:761-9.

7. Odeh M, Tendler R, Kais M, Maximovsky O, Ophir E, Bornstein J. Early pregnancy failure: factors affecting successful medical treatment. Isr Med Assoc J 2010; 12:325-8.

8. Ashok PW, Templeton A, Wagaarachchi PT, Flet GM. Factors affecting the outcome of early medical abor- tion: a review of 4132 consecutive cases. $\mathrm{Br} \mathrm{J}$ Obstet Gynaecol 2002;109:281-9.

9. Robledo C, Zhang J, Troendle J, Barnhart K, Creinin MD, Westhoff C, Huang X, Frederick M. Clinical indicators for success of misoprostol treatment after early pregnancy failure. Int J Gynaecol Obstet 2007;99:4651.

10. The management of early pregnancy loss. Royal College of Obstetricians and Gynaecologists. Green-Top guideline 25. October 2006. 